\title{
A rede da Política Nacional de Agricultura Familiar no Brasil
}

\author{
LUCIANA NUNES GOULART ${ }^{1}$ \\ DIEGO MOTA VIEIRA ${ }^{2}$ \\ Daniela Matias de Carvalho Bittencourt ${ }^{3}$ \\ 1 Tribunal de Contas da União (TCU), BRAsília - DF, BRAsil \\ 2 Universidade de Brasília (UnB) / Programa de Pós-Graduação em AdMinistração, Brasílıa - DF, Brasil \\ ${ }^{3}$ Empresa Brasileira de Pesquisa Agropecuária (EMBRAPA) / Recursos Genéticos e Biotecnologia, Brasília - DF, Brasil
}

\begin{abstract}
Resumo
Este artigo apresenta uma análise de redes de políticas públicas com foco na Política Nacional de Agricultura Familiar no Brasil. O estudo coletou dados por meio de análise documental, consultas orçamentárias e entrevistas, submetendo as informações obtidas a análise de conteúdo e, com isso, identificou a composição da rede de atores envolvidos na gestão da política, bem como as características das relações estabelecidas entre eles. Esse tipo de análise constitui interessante instrumento para os estudos de implementação e de avaliação de políticas públicas, na medida em que evidencia de que modo as características da interação entre atores podem afetar o alcance dos objetivos das políticas públicas. A rede da Política Nacional de Agricultura Familiar existente à época da pesquisa se mostrava densa em seu centro e flexível em suas margens, ou seja, era formada por um importante conjunto de atores atuando de modo concomitante em diversos programas e outro conjunto de atores atuando de modo mais pontual ou localizado. Porém, tanto no centro quanto nas margens, observou-se que diversos atores nem sempre são tão atuantes quanto seria esperado. Assim, com base no referencial teórico de análise de redes de políticas públicas, sugere-se que tal situação compromete o funcionamento da rede e o resultado das políticas subjacentes. Ademais, as características da rede estudada demonstram a importância do pacto federativo para o funcionamento da Política Nacional de Agricultura Familiar e mostram que os gestores subnacionais devem ter seus interesses e demandas efetivamente considerados para que os resultados almejados sejam atingidos.
\end{abstract}

Palavras-chave: Redes de políticas públicas. Agricultura familiar. Relações em rede.

\section{Family farming policy network in Brazil}

\begin{abstract}
This paper presents a public policy network analysis carried out on the Brazilian National Family Farming Policy. The study collected data through documentary review, budget consultations, and interviews, submitting the obtained information to content analysis. The results identified the constitution of the network of actors involved in policy management, as well as the characteristics of the relationships established among them. This type of analysis is an important instrument for studies on the implementation and evaluation of public policies, as it shows how the characteristics of interactions between actors can affect the success of public policies. The National Family Farming Policy network, at the time of the research, was dense at its center and flexible at its margins, meaning that it was formed by an important set of actors concurrently working in several programs and another set of more localized actors. Nonetheless, both at the center and at the margins, it was observed that several actors are not always as active as expected. Based on the public policy networks analysis theoretical framework, it is suggested that such a situation compromises the network's functioning and the outcome of the underlying policies. Moreover, the network characteristics reinforce the importance of the federative pact for the functioning of family farming policy in Brazil and show that the interests and demands of subnational managers should be effectively considered to achieve the expected results.
\end{abstract}

Keywords: Public policy networks. Family farming. Network relationships.

\section{La red de la Política Nacional de Agricultura Familiar en Brasil}

\section{Resumen}

Este artículo presenta un análisis de redes de políticas públicas enfocado en la Política Nacional de Agricultura Familiar de Brasil. El estudio recolectó datos a través del análisis documental, consultas presupuestarias y entrevistas, sometió la información a análisis de contenido y, de esa manera, identificó la composición de la red de actores involucrados en la gestión de la política, así como las características de las relaciones establecidas entre ellos. Este tipo de análisis es un instrumento interesante para estudios de implementación y de evaluación de políticas públicas, ya que muestra cómo las características de interacción entre actores pueden afectar el logro de los objetivos de las políticas públicas. La red de la Política Nacional de Agricultura Familiar existente en el momento de la investigación era densa en su centro y flexible en sus márgenes, es decir, estaba formada por un conjunto de actores que operaban simultáneamente en varios programas y otro conjunto de actores que operaba de manera más puntual. Sin embargo, tanto en el centro como en los márgenes, se observó que varios actores no siempre fueron tan activos como se esperaba. Por lo tanto, con base en el marco teórico de análisis de redes de políticas públicas, se sugiere que esta situación compromete el funcionamiento de la red y el resultado de las políticas subyacentes. Además, las características de la red estudiada demuestran la importancia del pacto federativo para el funcionamiento de la Política de Agricultura Familiar brasileña y muestran que los intereses y demandas de los gestores subnacionales deben ser efectivamente considerados para lograr los resultados deseados.

Palabras clave: Redes de políticas públicas. Agricultura familiar. Relaciones en red. 


\section{INTRODUÇÃO}

Em sua clássica obra sobre implementação de políticas públicas, Pressman e Wildavsky $(1984$, p. 133) afirmaram que a mais frequente recomendação para a ação governamental era "precisamos de maior coordenação" e nenhuma reclamação sobre serviços públicos era tão frequente quanto a "falta de coordenação". De acordo com Jennings (1994), o conceito de coordenação se tornou um dos mais discutidos nas literaturas sobre organizações e políticas públicas, em função da natureza cada vez mais complexa das questões com que o setor público passou a se deparar (Ferlie, Fitzgerald, McGivern \& Dopson, 2011; O’Toole, 1997; Peters, 1998; Repetto, 2009).

De fato, é notável que a administração pública, com frequência, busque organizar-se a partir da atuação conexa de atores diversos, em contextos de interseções entre mandatos e nos quais a hierarquia tradicional tem pouco valor. Tais contextos de interação são denominados pela literatura redes de políticas públicas (O’Toole, 1997; Provan \& Milward, 1995).

Essas redes operam em contextos nos quais estão presentes incentivos tanto à cooperação quanto à ação isolada - principalmente quando diferentes programas e agências são criados ao longo do tempo para atender às necessidades similares ou até sobrepostas (Cline, 2000; Jennings \& Ewalt, 1998).

Considerando tal fato, este estudo analisou a composição e as relações da rede da Política Nacional de Agricultura Familiar no Brasil. Esta enfrenta importantes desafios de coordenação, o que torna adequada a aplicação da análise de redes sociais (ARS) que provê ferramentas para identificar aspectos como poder e capacidade de acessar recursos, por meio de características posicionais e relacionais de atores inseridos em um contexto institucional semiestável de relações de cooperação em busca de determinados objetivos (Burt, 1992; Borgatti, Everett \& Johnson, 2013; Fischer \& Sciarini, 2015; Leifeld \& Schneider, 2012).

Por entender que é crucial considerar as origens da agricultura do tipo familiar no país, de modo a compreender suas atuais necessidades e sua imensa complexidade social, este artigo se inicia com uma apresentação dos antecedentes históricos que levaram à formação da rede da Política Nacional de Agricultura Familiar no Brasil. Em seguida, descreve-se a metodologia que possibilitou identificar a composição e as características das relações da rede - apresentadas nas sessões posteriores. Por fim, apresentam-se algumas implicações dos achados de pesquisa e apontam-se possíveis soluções para o melhor funcionamento da rede analisada.

\section{ANTECEDENTES HISTÓRICOS DA POLÍTICA DE AGRICULTURA FAMILIAR NO BRASIL}

O Estatuto da Terra (Lei n. 4.504, 1964, art. 4으, II) definiu como propriedade familiar

[...] o imóvel rural que, direta e pessoalmente explorado pelo agricultor e sua família, Ihes absorva toda a força de trabalho, garantindo-lhes a subsistência e o progresso social e econômico, com área máxima fixada para cada região e tipo de exploração, e eventualmente trabalho com a ajuda de terceiros.

A definição trazida pelo Estatuto da Terra (Lei n. 4.504, 1964) foi a primeira legislação sobre o tema e mostra-se importante por ter delineado critérios de definição da propriedade familiar utilizados até os dias de hoje. No entanto, considera-se de fundamental importância retroceder ao momento anterior, para compreender a agricultura familiar enquanto fenômeno social, indo além de suas definições legais. Assim, o modelo de trabalho familiar rural no Brasil se originou ainda no período colonial, com as grandes propriedades e a subserviência dos trabalhadores do campo (Guanziroli, 1999; Moreira, 2009; Picolotto, 2014).

De acordo com Moreira (2009), o fato dos trabalhadores rurais brasileiros terem surgido em uma posição subalterna levou à sua estigmatização e à sua associação a imagens de estagnação e conformismo. Assim, o mercado de trabalho rural se originou de modo socioeconomicamente desvalorizado, relacionado apenas à agricultura de subsistência e à reserva de mão de obra (Picolotto, 2014) - na visão desse autor, isso ajuda a compreender porque somente em meados do século XX surgiram organizações de representação política dos grupos mais desfavorecidos do meio rural.

Já com o início da abertura política, durante a fase de democratização, o campo presenciou uma expressiva mobilização social. Houve certa aproximação entre o Estado e as organizações sociais rurais, visto que, já no governo Sarney, foi criado o Ministério da Reforma e do Desenvolvimento Agrário e foi apresentado o primeiro Plano Nacional de Reforma Agrária (Dias, 2007). 
Ademais, a Constituição Federal de 1988 equiparou o trabalhador rural ao urbano, para fins assistenciais e previdenciários, apesar de ter limitado as possibilidades de mudanças significativas na estrutura fundiária brasileira, mantendo o acesso à terra nos moldes definidos pelo Estatuto da Terra, de 1964 (Da Ros, 2006).

Pouco depois da Assembleia Constituinte foi publicada a Lei n. 8.171/1991, sobre política agrícola. Algumas das demandas dos pequenos produtores foram incorporadas por essa lei, que reconheceu a diversidade dos agricultores brasileiros e previu a construção de uma política agrícola diferenciada para os produtores de pequeno porte. No entanto, os avanços também ficaram aquém das reinvindicações, pois prevaleceram os interesses dos grandes setores agrícolas (Fernandes, 2016; Grisa \& Schneider, 2014; Picolotto, 2014).

No mesmo sentido, a Lei n. 8.629 (1993) regulamentou dispositivos constitucionais sobre reforma agrária e definiu os conceitos de propriedade produtiva e de pequena e média propriedade - que, em regra, não são sujeitas a desapropriações. Essa lei buscou desestimular invasões e conflitos fundiários por meio de punições significativas (Lei n. 8.629, 1993, art. 2ㅇ, §§ 6으 e 7ㅇ) e manteve boa parte da estrutura regulamentar prevista pelo Estatuto da Terra (Lei n. 4.504, 1964).

Ainda na década de 1990, desenvolveu-se um projeto de cooperação técnica entre o Instituto Nacional de Colonização e Reforma Agrária (Incra) e a Organização das Nações Unidas para a Alimentação e a Agricultura (Food and Agriculture Organization of the United Nations [FAO]), com o objetivo de produzir subsídios para uma Política Nacional de Agricultura Familiar. O estudo Incra/FAO identificou a categoria agricultor familiar como um grupo bastante heterogêneo, formado por 3 grandes subgrupos: a) periférico; b) de transição; e c) consolidado.

Os periféricos seriam os agricultores de baixa renda, que normalmente vivem em áreas muito pequenas. Para o modelo Incra/ FAO, tal grupo deveria ser alvo principalmente de políticas assistenciais, pois "apresenta poucas perspectivas de subsistência e desenvolvimento no contexto das políticas agrícolas e agrárias" (Guanziroli, 1999, p. 16). O grupo de transição, por sua vez, constituía-se de famílias com renda semelhante à renda média no Brasil e superior à dos trabalhadores agrícolas temporários. Já os produtores consolidados seriam aqueles com rendas altas, com capacidade de gerar seu próprio desenvolvimento, e, portanto, sem necessidade de apoio governamental direto (Guanziroli, 1999).

O estudo Incra/FAO assumiu grande importância, pois definiu quem deveria ser o público alvo prioritário do primeiro programa oficialmente voltado à agricultura familiar: o Programa Nacional de Fortalecimento da Agricultura Familiar (Pronaf) (Cazella et al., 2016; Guanziroli, 1999; Picolotto, 2014). Assim, o Pronaf surgiu para atender prioritariamente ao chamado grupo de transição, em função de seu significativo potencial de crescimento econômico e de consolidação (Guanziroli, 1999).

A heterogeneidade da categoria agricultor familiar coloca o poder público diante de um problema complexo, que exige que diferentes soluções sejam oferecidas aos diversos grupos, considerando, ainda, as peculiaridades regionais e o papel de estados e municípios. Nesse sentido, Moreira (2009) afirma que o uso da categoria geral agricultores familiares obscurece suas diferentes formas sociais, bem como as diferenças regionais. Nas palavras de Guanziroli (1999, p. 19):

Agricultores familiares e assentados constituem um público heterogêneo, diversificado e complexo, o que exige soluções diferenciadas. Há os posseiros, os parceiros, os arrendatários, os trabalhadores de tempo parcial, os boias frias, os assalariados permanentes, os temporários, os desempregados do campo, os filhos dos pequenos proprietários, os minifundistas, os próprios assentados da reforma agrária e os agricultores familiares consolidados. A proposta não pode ser igual para todos. Alguns já possuem terra, como os minifundistas, porém[,] de tamanho reduzido, outros têm a posse precária, como os posseiros e os parceiros, outros precisam basicamente de infraestrutura.

O processo no qual uma rede de atores se envolveu para criar um programa específico para a agricultura familiar foi descrito por Navarro (2010). O autor relata que o movimento tomou corpo quando, no contexto da implementação do Mercado Comum do Sul (Mercosul)1', pequenos produtores uruguaios, paraguaios e brasileiros, sentindo-se excluídos das negociações, alianharam-se em busca de suporte governamental. Foi nesse contexto que a "agricultura familiar, como expressão da agenda nacional, adentrou o cenário político" (Navarro, 2010, p. 192).

Segundo Navarro (2010), coube à Confederação Nacional dos Trabalhadores da Agricultura (Contag) a liderança desse processo. A Contag se tornou interlocutora legítima das discussões com o então Ministério da Agricultura, Abastecimento e Reforma

${ }^{1}$ Criado pelo Tratado de Assunção, de 1991 
Agrária (Maara) (Caume, 2009; Navarro, 2010). Com a aproximação, o Maara editou a Portaria Ministerial n. 692/1993, criando um grupo de trabalho com participação da Contag. Esse grupo apresentou uma proposta, assinada pelo Presidente da República em 1994, denominada Programa de Viabilização da Pequena Propriedade, que permitiu a criação do Pronaf (Navarro, 2010).

Durante esse processo, o governo reconheceu a categoria agricultura familiar como detentora de necessidades a ser atendidas por políticas públicas. Assim, foram criados, em 1999, o Conselho Nacional de Desenvolvimento Rural Sustentável (CONDRAF) e o Ministério da Política Fundiária e Agricultura Familiar, que, em 2000, passou a ser denominado Ministério do Desenvolvimento Agrário (MDA) (Caume, 2009).

Vale notar que o contexto no qual o programa se originou foi responsável por definir o enfoque de atuação que até hoje caracteriza o Pronaf, que opera pela concessão de financiamentos subsidiados a projetos produtivos. Tal como formatado, o programa atende de modo bastante adequado aos agricultores do Sul do país, que foram os atores que lograram mobilizar-se durante sua criação, por terem sido os mais afetados pela criação do Mercosul. Assim, ainda hoje a política tem características que refletem o contexto de sua formação.

Após a consolidação do Pronaf foram criados diversos outros programas voltados à agricultura familiar, ao longo dos anos 2000. Ademais, aprovou-se a Lei n. 11.326/2006, que definiu a categoria agricultor familiar e estabeleceu outras diretrizes. Atualmente, existem vários atores envolvidos na gestão de diversos programas, caracterizando uma verdadeira rede de política pública, nos termos definidos por Provan e Milward (1995): são membros da rede todas as organizações - governamentais ou não - que prestam serviços públicos a determinado público-alvo.

Além dos membros, este estudo também levantou os stakeholders externos da rede, entendidos como aquelas organizações que, apesar de não proverem diretamente serviços públicos ao público-alvo, têm relações de influência relevantes sobre a rede e devem receber atenção por parte de seus gestores, a partir de uma avaliação de atributos de poder, legitimidade e urgência, segundo Mitchell, Agle e Wood (1997).

\section{METODOLOGIA}

Isso posto, passa-se a uma breve descrição da metodologia utilizada para identificar membros e stakeholders externos e as características das relações que esses atores estabeleceram no âmbito da rede.

Para tanto, iniciou-se com a análise documental de leis, decretos, planos e outros documentos oficiais que definem atribuições dos membros da rede e/ou que apresentam ligações relevantes com stakeholders. Um tipo de documento que se mostrou bastante útil para a identificação de relações de rede, por evidenciar a atuação e as relações entre os diversos atores, foram os Espelhos de Monitoramento de 2018 do Plano Plurianual, disponíveis no Sistema Integrado de Planejamento e Orçamento (SIOP). Esse documento é rico em informações prestadas pelos próprios gestores e apresenta suas principais conquistas e dificuldades de execução.

Ademais, realizaram-se consultas orçamentárias a 51 ações voltadas à agricultura familiar, com vistas a confirmar as unidades orçamentárias e as unidades gestoras responsáveis pela execução de recursos para esse público-alvo². Paralelamente, analisou-se a composição de diversos fóruns relevantes ${ }^{3}$ e realizaram-se entrevistas em profundidade, com roteiro semiestruturado, junto a atores-chave dentro e fora do governo.

Por oportuno, agregam-se algumas informações sobre essas entrevistas. Primeiro, realizou-se entrevista piloto, em 29 de junho de 2018, com um dos principais gestores da política nacional à época. Posteriormente, realizaram-se outras 24 entrevistas no período entre 18 de outubro de 2018 e 19 de dezembro de 2018, com um total de 29 gestores ou stakeholders. Todas as entrevistas, com duração média de 1 hora, foram gravadas e transcritas na íntegra.

\footnotetext{
${ }^{2}$ As consultas orçamentárias foram realizadas no Sistema Integrado de Administração Financeira do Governo Federal (SIAFI), sobre a execução dos exercícios de 2013 a 2018, por meio do site do Tesouro Gerencial.

${ }^{3}$ Conselho Nacional de Desenvolvimento Rural Sustentável (CONDRAF); Conselho Nacional de Segurança Alimentar e Nutricional (Consea); Conselho Nacional de Agroecologia e Produção Orgânica (CNAPO); Comitê Gestor do Água para Todos e do Garantia-Safra; Grupos Consultivos do Programa de Aquisição de Alimentos (PAA) e do Programa Nacional de Alimentação Escolar (PNAE); e Conselho de Administração da Agência Nacional de Assistência Técnica e Extensão Rural (Anater) e Conselho Assessor da Anater.
} 
As entrevistas foram submetidas a análise de conteúdo, assim como os demais documentos utilizados na pesquisa, seguindo a metodologia proposta por Bardin (2009), que possibilita a categorização sistemática do conjunto de dados (categorias estabelecidas a priori, com base no referencial teórico e nos objetivos da pesquisa), de modo a facilitar a apreensão das informações relevantes, tornando-as pertinentes em relação ao tema.

De posse das informações obtidas, procedeu-se ao registro das relações envolvidas em cada um dos principais programas públicos federais voltados à agricultura familiar, por meio da metodologia da ARS (Burt, 1992; Borgatti et al., 2013; Fischer \& Sciarini, 2015; Leifeld \& Schneider, 2012). Foi identificado um total de 30 programas ou ações de relevo, cujas relações subjacentes foram documentadas e processadas via software Gephi (The Gephi Consortium, c2017), uma plataforma livre que possibilita a visualização de grafos dinâmicos que auxiliam no entendimento do formato de redes, por meio do cálculo automático e da interpretação de métricas (Bastian, Heymann \& Jacomy, 2009). O uso do software viabilizou a obtenção das estatísticas de densidade de rede, de posição e de centralidade de seus membros (Blondel, Guillaume, Lambiotte \& Lefebvre, 2008; Brandes, 2001; Brin \& Page, 1998).

Vale esclarecer que os resultados aqui apresentados refletem a rede existente em finais de 2018, período de execução desta pesquisa. As alterações trazidas pelo governo Bolsonaro, por meio da Medida Provisória n. 870/2019, ocorreram quando já havia sido finalizada a fase de coleta de dados, ficando, portanto, fora do escopo analisado. Porém, considerando que os programas avaliados apresentaram alterações apenas em suas titularidades, considera-se que a perda analítica decorrente é pequena, uma vez que não é difícil reposicionar os atores relevantes na nova estrutura.

\section{COMPOSIÇÃO DA REDE DA POLÍTICA NACIONAL DE AGRICULTURA FAMILIAR}

Com a aplicação da metodologia descrita foram identificados 171 participantes, entre membros e stakeholders externos da rede da Política Nacional de Agricultura Familiar. A Tabela 1 sumariza essa composição.

Tabela 1

Composição da rede da Política Nacional de Agricultura Familiar

\begin{tabular}{ccccc} 
& Governo & $\begin{array}{c}\text { Sociedade civil/ } \\
\text { setor privado }\end{array}$ & Total & Percentual \\
\hline Membros & 65 & 11 & 76 & $44,4 \%$ \\
\hline Stakeholders externos & 28 & 67 & 95 & $55,6 \%$ \\
\hline Total & 93 & 78 & 171 & $100 \%$ \\
\hline Percentual & $54,4 \%$ & $45,6 \%$ & & \\
\hline
\end{tabular}

Fonte: Elaborada pelos autores.

Na realidade, o número de organizações participantes é muito maior. Isso porque "governos municipais", por exemplo, foram tratados como uma única entidade, visto que seus interesses e atribuições em relação à rede nacional podem ser considerados relativamente homogêneos. Também pelo mesmo motivo, "entidades privadas de ATER" foram tratadas como uma única organização fictícia que, na verdade, representa centenas de empresas espalhadas pelo país ${ }^{4}$, dentre outros casos. Assim, os 171 participantes representam, em alguns casos, organizações de fato e, em outros, tipos de organizações, o que reforça a complexidade da rede real.

De toda forma, mesmo com essas simplificações, indentificou-se uma rede bastante grande. Esse é um aspecto relevante, visto que a literatura aponta que há um limite para o número de ligações que determinada organização consegue gerenciar efetivamente (Mitchell \& Shortell, 2000; Provan \& Kenis, 2007; Provan \& Milward, 1995). Assim, mesmo ponderando que os atores não estão conectados a todos os demais na prestação dos serviços, o elevado número de membros e de stakeholders externos é um indicativo de que as organizações estão incorrendo em altos custos de gerenciamento (Berardo, 2009;

${ }^{4}$ Em 27 de janeiro de 2019 havia 316 empresas credenciadas pela Anater, conforme consulta ao Sistema de Gestão de ATER. 
Leifeld \& Schneider, 2012). Ademais, redes muito grandes apresentam maior tendência à sobreposição de atividades (Raab, Mannak \& Cambré, 2013), o que compromete a eficiência e a economicidade do gasto público.

Após identificada a composição da rede, passa-se a uma breve análise de seu grau de heterogeneidade. De acordo com Mitchell e Shortell (2000), uma rede é tanto mais heterogênea quanto mais possuir atores do setor privado e da sociedade civil em sua composição. Para os autores, quanto mais complexos os problemas a ser atacados pela rede, maior deve ser seu nível de heterogeneidade, pois, com a presença de atores de diferentes tipos, torna-se possível sopesar legitimidade e alcance de resultados (Mitchell \& Shortell, 2000).

No caso da rede da Política Nacional de Agricultura Familiar, o recorte da Tabela 1 já possibilita afirmar que se trata de uma rede bastante heterogênea, de variância igual a 0,248 , muito próxima à variância máxima para variáveis binárias $(0,25)^{5}$.

No entanto, considerando a especificidade do caso, entende-se pertinente diferenciar, dentre os atores não governamentais, as organizações representantes da agricultura familiar e outras organizações privadas, como mostra a Tabela 2.

Tabela 2

Destaque para as organizações que representam a agricultura familiar na composição da rede

\begin{tabular}{cccc}
\hline Governo & $\begin{array}{c}\text { Atores privados } \\
\text { diversos }\end{array}$ & $\begin{array}{c}\text { Organizações representantes } \\
\text { da agricultura familiar }\end{array}$ & Total \\
\hline 93 & 43 & 35 & 171 \\
\hline $54,4 \%$ & $25,15 \%$ & $20,45 \%$ & $100 \%$ \\
\hline
\end{tabular}

Fonte: Elaborada pelos autores.

Assim, percebe-se que as organizações representantes da agricultura familiar respondem por menos de $21 \%$ dos participantes identificados por esta pesquisa ${ }^{6}$, o que pode significar menores ganhos de legitimidade em decorrência da heterogeneidade geral da rede (Mitchell \& Shortell, 2000).

Adicionalmente, e considerando o importante papel desempenhado pelo CONDRAF, de "propor diretrizes para a formulação, a implementação e a execução de políticas públicas estruturantes voltadas para o desenvolvimento rural sustentável, a reforma agrária, o reordenamento fundiário e a agricultura familiar" (Decreto n. 9.186, 2017, art. 10)7, analisou-se a evolução da composição desse conselho desde sua criação, em relação à participação de atores governamentais e de organizações representantes da agricultura familiar ${ }^{8}$.

O CONDRAF foi criado pelo Decreto n. 3.200/1999 e passou por alterações determinadas pelos Decretos n. 3.508/2000; $3.992 / 2001 ; 4.854 / 2003 ; 8.735 / 2016$ e 9.186/2017. Os gráficos que se seguem foram elaborados com base nessas normativas. O primeiro deles apresenta, a cada composição vigente, apenas membros com direito a voto.

Na Figura 1 se percebe que a participação de organizações representantes da agricultura familiar, dentre os membros do CONDRAF, foi aumentando até atingir seu ápice com o Decreto n. 8.735/2016. Vale destacar que a composição cristalizada por essa normativa foi fruto de um estudo de recomposição do conselho desenvolvido por um grupo de trabalho ${ }^{9}$ formado para

\footnotetext{
${ }^{5}$ A variância máxima para variáveis binárias é obtida pela multiplicação dos percentuais das 2 categorias, no caso: 0,456 (não governamental) x 0,544 (governamental) $=0,248$.

${ }^{6}$ É possível que haja outras, dos diversos tipos, que não apareceram nesta estratégia de coleta de dados.

${ }^{7}$ O Decreto n. 9.186/2017 foi revogado pelo Decreto n. 9.759/2019, que extinguiu 55 órgãos colegiados do Governo Federal, inclusive o CONDRAF.

${ }^{8}$ Nesta análise foram desconsideradas organizações que, embora não fossem exatamente governamentais, tinham forte ligação formal com o governo e/ou que, mesmo representando a sociedade civil, tampouco poderiam ser consideradas representantes da agricultura familiar. As organizações desconsideradas para fins da análise apresentada nos gráficos às figuras 1 e 2 foram, ao longo de todo o período: Fórum Nacional dos Secretários da Agricultura, Serviço Brasileiro de Apoio às Micro e Pequenas Empresas (Sebrae), Associação Nacional dos Órgãos Estaduais de Terra (ANOTER), associações de municípios, Associação Brasileira das Entidades Estaduais de Assistência Técnica e Extensão Rural (Asbraer), Conselho Nacional das Entidades Estaduais de Pesquisa Agropecuária (Consepa) e Conselho Nacional dos Secretários de Estado de Agricultura (CONSEAGRI), que são órgãos não governamentais, mas que têm fortes ligações formais com o governo. Também foram desconsiderados o Conselho Nacional de Segurança Alimentar e Nutricional (Consea) e a Comissão Nacional de Agroecologia e Produção Orgânica (CNAPO), que, embora constituíssem instituições nas quais havia uma grande influência dos interesses da agricultura familiar, eram órgãos colegiados diretamente vinculados ao governo.

${ }^{9}$ Formado por representantes de Confederação Nacional dos Trabalhadores Rurais Agricultores e Agricultoras Familiares (CONTAG), Confederação Nacional dos Trabalhadores e Trabalhadoras na Agricultura Familiar do Brasil (Contraf Brasil), Conferência Nacional dos Bispos do Brasil (CNBB), Articulação Semiárido Brasileiro (ASA), Rede dos Centros de Formação por Alternância e Ministério do Desenvolvimento Agrário (MDA), conforme ata da 60ā Reunião Ordinária do CONDRAF, de 13 e 14 de maio 2015.
} 
propor uma estrutura mais atualizada às necessidades da agricultura familiar da época. No entanto, a composição definida pelo decreto de 2016 não foi colocada em prática, já que o conselho ficou paralisado por 2 anos, após o impeachment da presidente Dilma Rousseff. Já no governo Michel Temer, e antes que o conselho voltasse a se reunir, foi estabelecida uma nova composição, pelo Decreto n. 9.186/2017, na qual a participação se tornou paritária, com 22 membros de cada um dos grupos.

Figura 1

\section{Composição do CONDRAF desde sua criação - apenas membros (com direito a voto)}

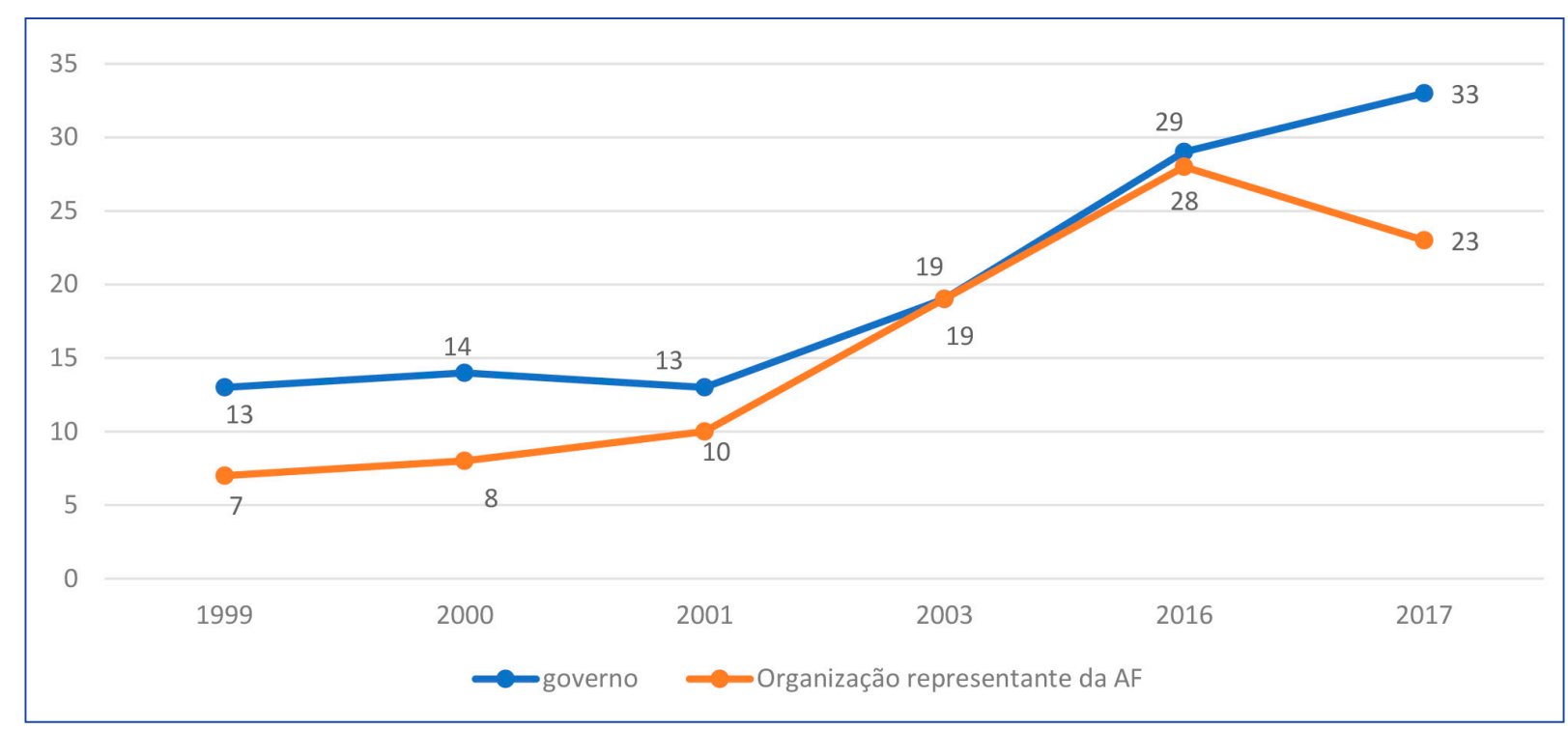

Fonte: Elaborada pelos autores.

No entanto, segundo o Entrevistado 15, a composição formada pelo decreto de 2017 não seria de fato paritária, em função da existência de uma série de organizações convidadas que, em sua maioria, são governamentais. Segundo esse entrevistado, apesar de não terem direito a voto, os convidados participam das discussões igualmente, podendo desbalanceá-las. Sendo assim, também se elaborou a Figura 2 que apresenta a evolução do CONDRAF ao longo do tempo, considerando, ainda, a presença de convidados (sem direito a voto).

Figura 2

Composição do CONDRAF desde sua criação - convidados incluídos

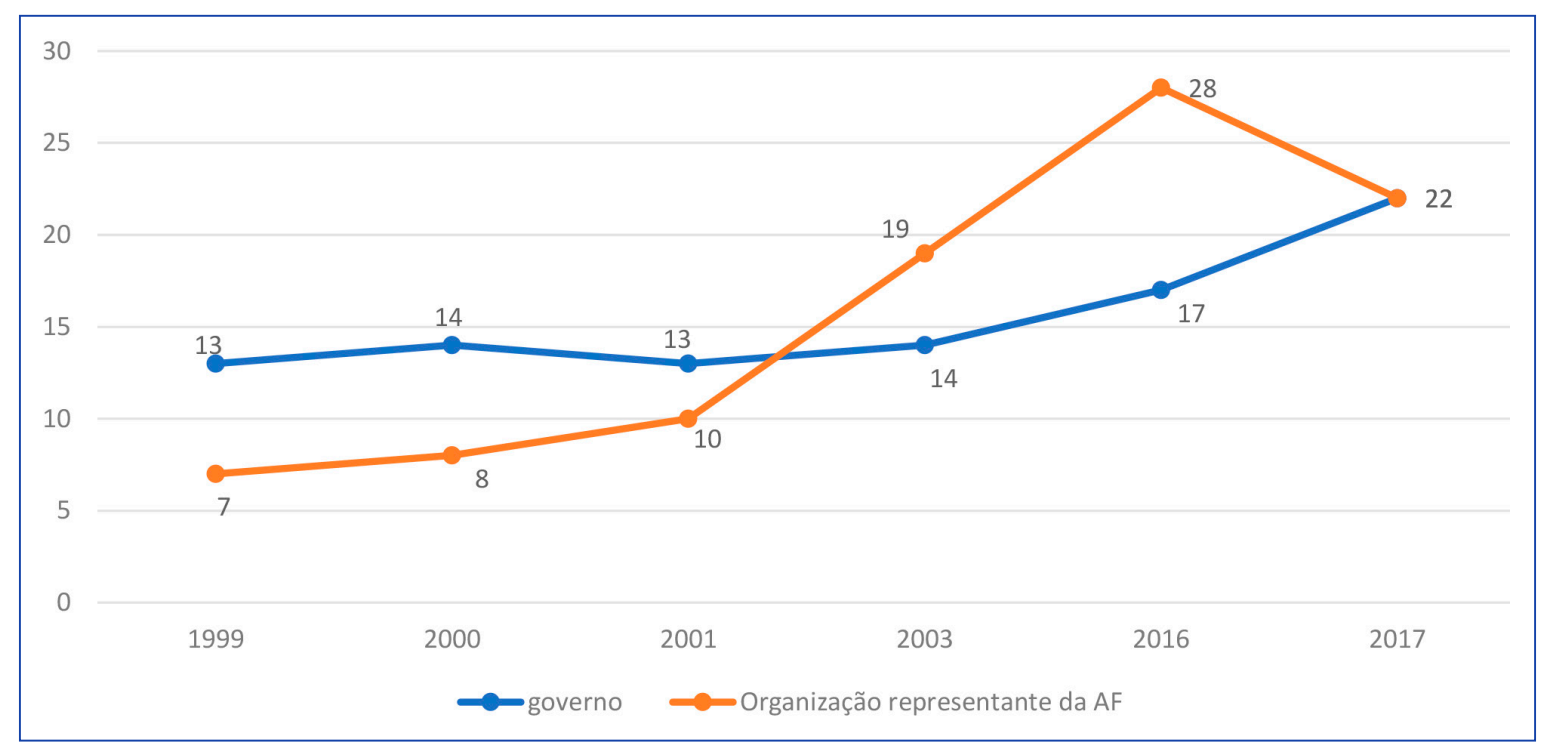

Fonte: Elaborada pelos autores. 
Assim, quando as organizações convidadas são consideradas, percebe-se uma predominância do governo em todo o período, com mais equilíbrio entre 2003 e 2016, mas com grande diferença na composição vigente à época da pesquisa.

Além dessas constatações, um último ponto acerca da composição da rede deve ser destacado. Segundo a literatura, deve ser avaliaado se todos os participantes relevantes estão incluídos na rede, pois quanto mais aderente sua composição, maior sua capacidade de obter recursos (Mandell \& Keast, 2007; Mitchell \& Shortell, 2000).

Conforme percepção dos diversos entrevistados, a composição da rede era adequada, apesar da representatividade de alguns setores ser frágil e o comprometimento dos diversos atores ser variável. Segundo o Entrevistado 24,

[...] a rede abarca meio que os atores adequados [...] é difícil você pensar em alguém que está sem palavra total, sem capacidade de verbalização.

Ou, nas palavras do Entrevistado 29,

[...] o problema não é que as organizações não estão presentes, é que a atuação deles é deficiente.

Inclusive, como ressaltado pelo Entrevistado 2, também era adequada a participação de grupos de interesse minoritários (p. ex., mulheres, jovens, comunidades tradicionais).

\section{CARACTERÍSTICAS DAS RELAÇÕES NA REDE}

Com o uso do software Gephi (The Gephi Consortium, c2017), elaboraram-se grafos que representam esquematicamente a rede da Política Nacional de Agricultura Familiar. A Figura 3 evidencia os programas e seus principais atores. A Figura 4 apresenta as relações entre os atores, tendo como base os programas nos quais esses atores atuam conjuntamente. E a Figura 5 a presenta as relações entre os programas, com base em seus atores em comum.

As diferentes cores nos grafos representam a formação de conglomerados, ou seja, grupos com características internas homogêneas e diferentes em relação aos demais. Nos grafos, fazer parte de um mesmo conglomerado indica pertinência temática entre os programas e/ou aponta a atuação conjunta de atores. A proximidade entre atores também indica a intensidade de suas conexões nos diversos programas. Já o diâmetro dos nós indica a centralidade dos atores e/ou programas, medida esta fortemente relacionada a seu grau de impotância na rede (Berardo, 2009; Fischer \& Sciarini, 2015; Leifeld \& Schneider, 2012).

O software Gephi (The Gephi Consortium, c2017) também retorna as estatísticas que suportam a construção dos grafos. Neste artigo, por questões de concisão, apenas algumas dessas estatísticas são destacadas, iniciando-se pelas densidades obtidas.

A literatura sobre redes de políticas públicas aponta que, em redes densas e bem integradas, os serviços oferecidos tendem a ser construídos de modo complementar, para que todo o leque de necessidades seja coberto, sem ocorrência de sobreposições. Ademais, quanto mais integrado for determinado membro da rede, maior tende a ser o nível de importância atribuído à rede por esse ator (Provan \& Milward, 1991, 1995).

A rede de programas e atores (Figura 3) apresenta densidade 0,019, a rede de atores (Figura 4) apresenta densidade 0,269 e a rede de programas (Figura 5) apresenta densidade 0,979. A alta densidade da rede de programas indica que a Política Nacional de Agricultura Familiar apresenta um grupo central de atores que agem em forte parceria e de modo concomitante em muitos programas, enquanto que as baixas densidades obtidas nas representações que consideram a participação dos atores (Figuras 3 e 4) indicam que o grupo central é acompanhado por um grande grupo de atores que tem atuação específica, localizada e/ou dispersa. Assim, como caracterizam Provan e Lemaire (2012), trata-se de uma rede integrada em seu centro e flexível em suas margens, o que em tese favorece a articulação e a capacidade da rede para gerar bons resultados.

Relativamente ao grafo da Figura 3, que apresenta as relações entre programas e atores, destacam-se as medidas de "grau de entrada" e de "grau de saída" (Borgatti, 2005). O maior índice de "grau de entrada" foi obtido nas ações de promoção da agroecologia, seguidas por: a) projetos públicos de irrigação (PPI); b) programas de regularização fundiária; c) Programa de Aquisição de Alimentos (PAA); e d) gestão de reservas extrativistas (RESEX), o que significa que mais atores participam na operacionalização desses programas. Já o maior "grau de saída" foi apresentado pelos governos estaduais, seguidos pela Secretaria Especial de Agricultura Familiar e do Desenvolvimento Agrário (SEAD), pelo Incra, por organizações representantes 
da agricultura familiar e pelo Ministério do Desenvolvimento Social (MDS), significando que esses atores participavam na maior quantidade de programas avaliados. Esse índice, assim como outros descritos abaixo, evidencia a importância dos governos estaduais para a execução dos programas voltados à agricultura familiar.

Já na Figura 4, que representa as relações entre os atores, esses 5 membros também apresentaram os maiores índices page ranks, uma medida qualificada da importância das ligações que considera não apenas a quantidade de ligações de determinado ator, mas também a relevância dessas conexões (Brin \& Page, 1998).

Ainda na Figura 4, quanto à medida de "grau ponderado", mais uma vez, a importância dos governos estaduais se destaca. Esse foi o ator que obteve o maior valor (grau $=121$ ), o que significa que esse é o ator que mais estabelece parcerias com outros atores dentro da rede da Política Nacional de Agricultura Familiar. Os governos estaduais são seguidos por: a) organizações representantes da agricultura familiar (grau $=114$ ); b) SEAD (grau = 107); c) Incra (grau = 104); e d) governos municipais (grau $=103$ ). A grande quantidade de atores envolvidos é um indicador da complexidade do ambiente no qual esses atores operam e dos altos custos de transação envolvidos (Leifeld \& Schneider, 2012; Mitchell \& Shortell, 2000).

Figura 3

Grafo da rede da Política Nacional de Agricultura Familiar (atores e programas)

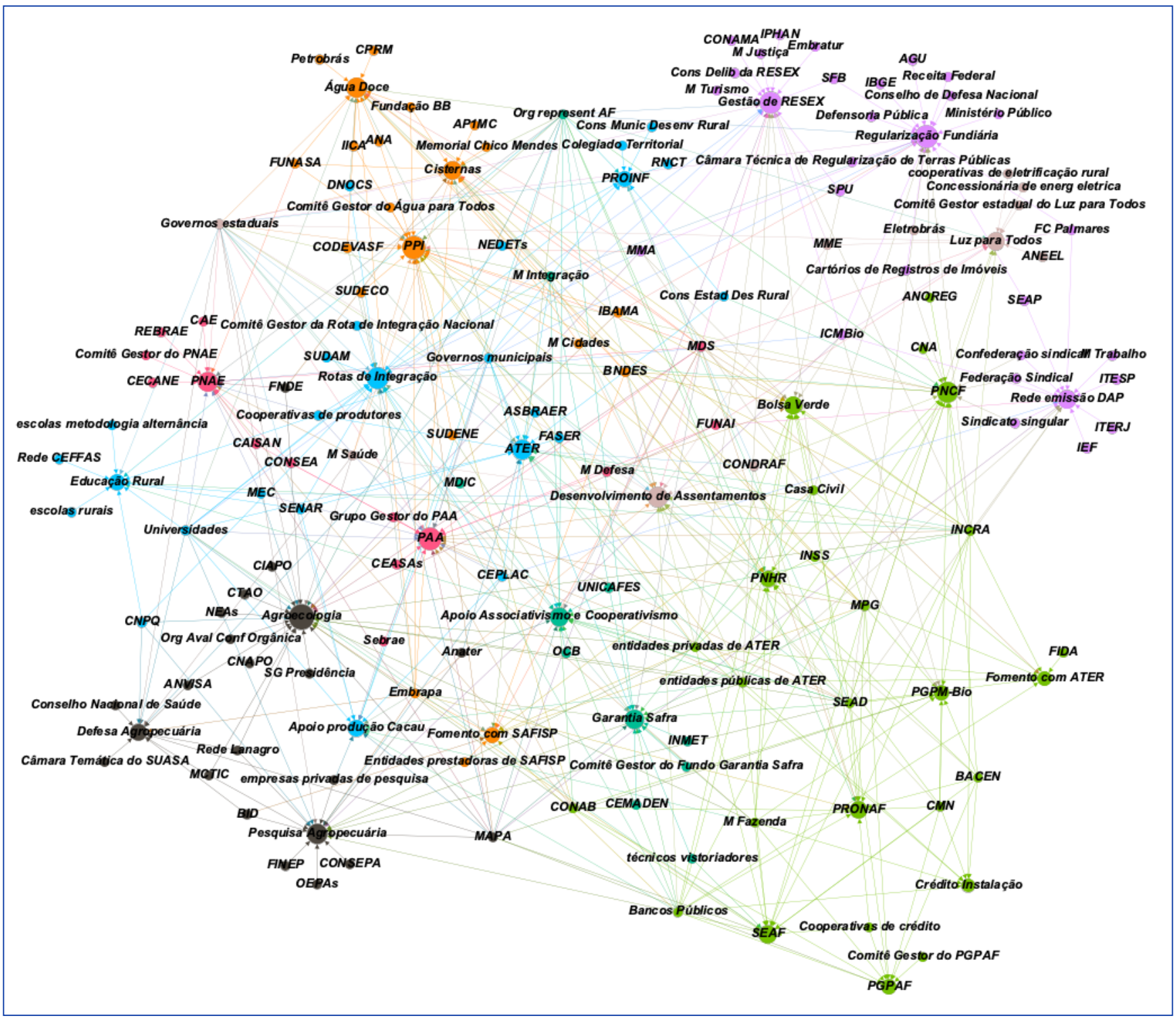

Fonte: Elaborada pelos autores. 
Figura 4

Grafo da rede de Atores da Política Nacional de Agricultura Familiar

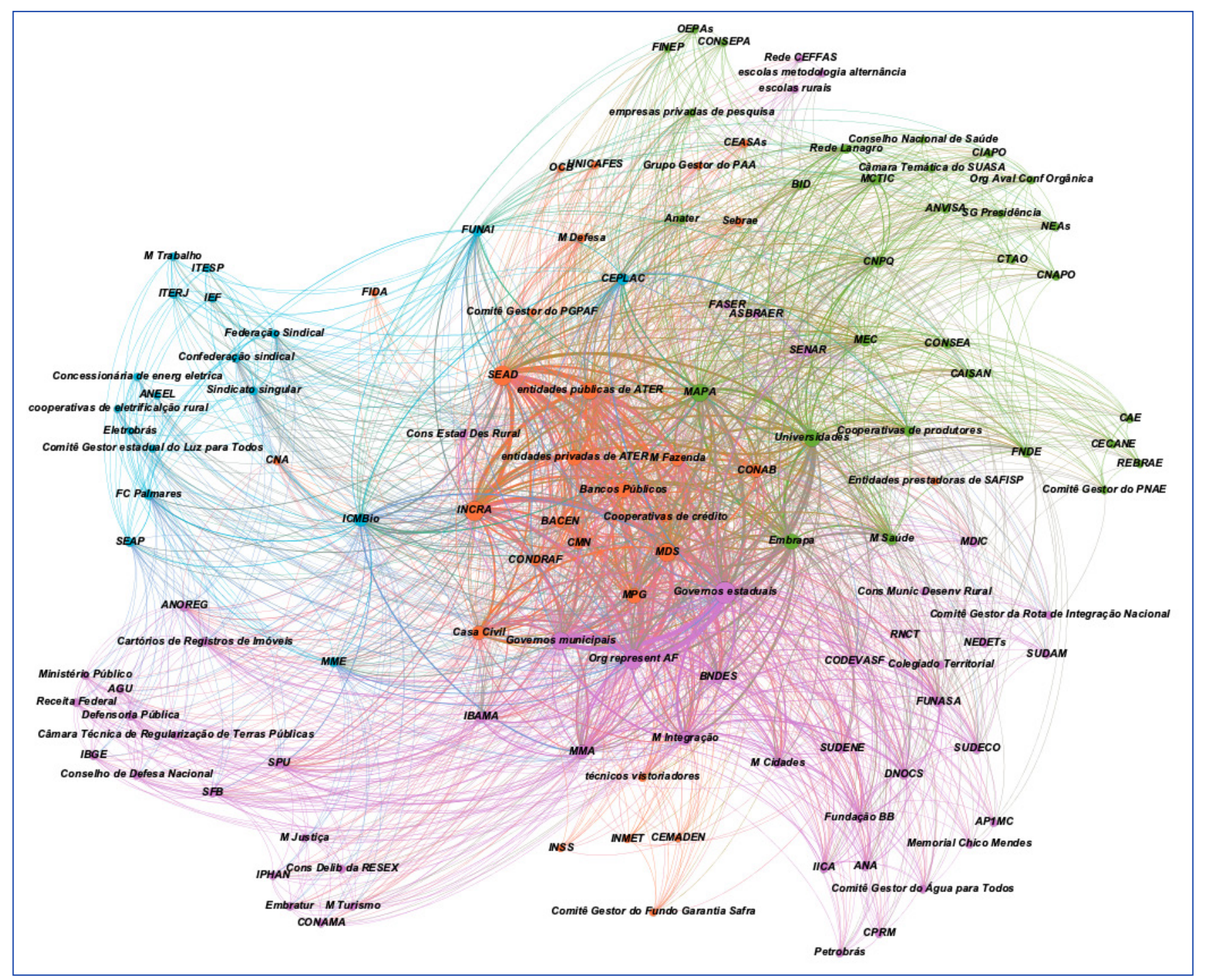

Fonte: Elaborada pelos autores.

Esses também foram os atores que apresentaram os maiores graus de centralidade de proximidade (CP) e de centralidade de intermediação $(\mathrm{Cl})$, a saber: a) governos estaduais ( $\mathrm{CP}=0,941 ; \mathrm{Cl} 0,084)$; b) organizações representantes da agricultura familiar ( $C P=0,895 ; C l 0,067) ; c)$ SEAD ( $C P=0,854 ; C l=0,067)$; $d$ Incra $(C P=0,837 ; C l=0,051)$; e e) governos municipais $(C P=0,832 ; \mathrm{Cl}=0,052)$. A alta centralidade de proximidade de um ator indica que a distância média dos seus caminhos mais curtos até alcançar todos os demais atores é menor. Já a centralidade de intermediação indica a frequência com que esse ator está presente no caminho mais curto entre dois outros atores. Medidas de centralidade indicam os vértices mais importantes, com maior capacidade de influenciar o fluxo de recursos e informações. Por exemplo,atores com altas centralidades de intermediação tendem a ser capazes de ativar os vazios estruturais de uma rede (Burt, 1992), podendo, inclusive, promover a ligação entre conglomerados isolados.

Quanto ao grafo representado na Figura 5, que explora a relação dos programas por meio de seus atores, destaca-se que todos os programas obtiveram altos graus de integração (dos 30 programas analisados, 20 apresentaram o grau máximo possível de relações nessa rede, que equivale ao número de programas menos 1, ou seja, 29). Isso significa que esses 20 programas têm ao menos um ator em comum com todos os demais programas analisados (Borgatti, 2005). 


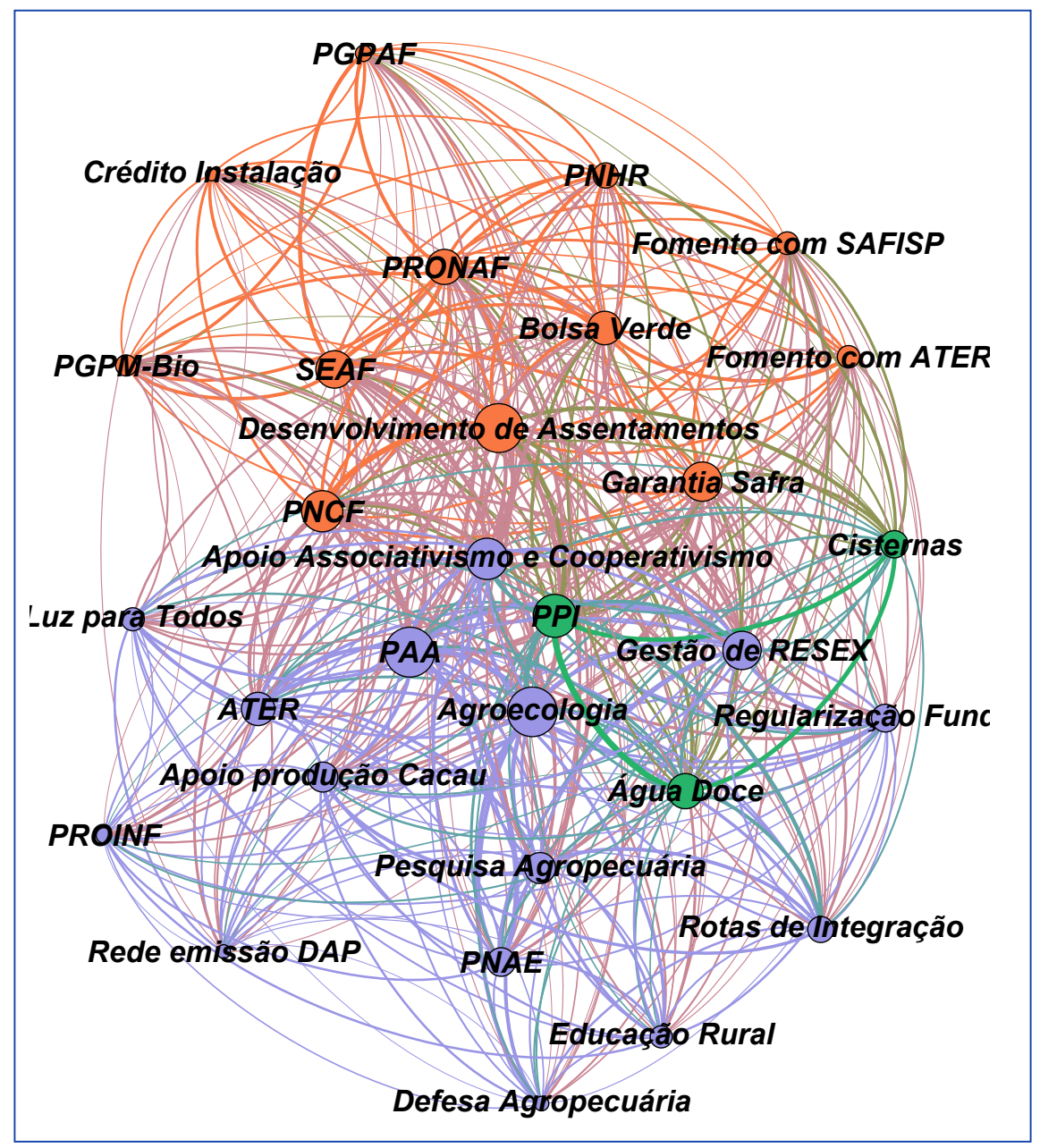

Fonte: Elaborada pelos autores.

Outra medida que se destaca no grafo 5 é a densidade de vizinhança (clustering) (Barrat, Barthelemy, Pastor-Satorras \& Vespignani, 2004; Borgatti et al., 2013). A vizinhança de um vértice representa o conjunto de todos os demais vértices ligados a ele. Assim, se todos os vizinhos de determinado vértice também forem ligados entre si, a densidade de vizinhança será igual a 1. Segundo Berardo (2009), altas densidades de vizinhança são um ativo importante, principalmente para vértices não centrais, isso porque as múltiplas ligações entre o grupo aumentam o custo reputacional da não colaboração, fazendo com que o ambiente tenda a ser mais cooperativo.

O Programa de Cisternas obteve densidade de vizinhança igual a 0,994, o que significa que praticamente todos os programas que têm atores em comum com o Programa de Cisternas também possuem atores comuns entre si. Obtiveram-se, ainda, altos índices de densidade de vizinhança os programas: a) Crédito de Instalação (0,993); b) Defesa Agropecuária (0,99); c) Programa Nacional de Alimentação Escolar (PNAE) (0,986); e d) Programa Nacional de Desenvolvimento Sustentável de Territórios Rurais (PROINF) (0,986).

Após apresentadas as representações da rede, e suas principais estatísticas, mostra-se fundamental explicitar a principal limitação desta análise, qual seja: o fato de que não foi possível mensurar de modo objetivo a intensidade da participação efetiva dos diversos atores. Pôde-se observar que todos os órgãos com atribuições legais nos programas sempre exercem tais atribuições, em alguma medida. Ou seja, não se pôde identificar nenhum ator faltante, que deveria estar atuando e não estava. 
No entanto, principalmente a partir das entrevistas e dos documentos analisados, percebeu-se que a intensidade da atuação de cada ator varia enormemente. Assim, o que ocorre, muitas vezes, é a participação distante de determinado ministério, ou a presença ativa de certo ator em alguns pontos do território, acompanhada por sua virtual ausência em outros. Desse modo, a atuação dos atores não é homogênea dentro dos diferentes programas. Eles não estão sem exercer suas atribuições, mas, muitas vezes, eles não estão tão presentes quando isso seria esperado para o bom andamento dos programas.

De todo modo, entendeu-se que não se dispunha de evidências suficientes para fazer refletir, nos grafos da rede, a intensidade da atuação real de cada um dos atores envolvidos, mostrando quem é mais ou menos atuante na prática. Diante disso, optou-se por apresentar a rede com base nas atribuições legais definidas para os programas, uma vez que tais atribuições estavam respaldadas pelas execuções orçamentárias apresentadas pelo Sistema Integrado de Administração Financeira do Governo Federal (SIAFI), pelas informações do acompanhamento da execução dos programas constantes dos Espelhos de Monitoramento do Plano Plurianua ${ }^{10}$ ou por entrevistados relevantes.

Desse modo, diante de evidências de que determinado membro de fato atua em um programa específico, sua participação nesse programa foi considerada, mesmo naqueles casos em que existiam indicativos de que essa participação estava fragilizada.

Reconhece-se a limitação desta análise, porém, optou-se por não atribuir valores à intensidade de participação de nenhum ator, visto que tal atribuição seria realizada de modo altamente subjetivo, principalmente em função da questão da abrangência territorial. Assim, mensurar o grau de participação em nível nacional não seria tarefa trivial e necessitaria de detalhamentos dos dados que não puderam ser obtidos.

No entanto, para buscar obter alguma aproximação com o real nível de integração dos atores na rede, procurou-se identificar nas entrevistas qual era a percepção dos entrevistados em relação ao tema. Nesse sentido, o nível de integração da rede, como apresentado pelos entrevistados, está bastante distante do que as atribuições legais preveem. De modo geral, na visão dos entrevistados, a articulação dos atores refletida na entrega de serviços é frágil.

Diversos entrevistados apontaram casos de sucesso para iniciativas localizadas, muitas vezes dependentes de empenho de pessoas específicas, e outros casos que resultam na implementação dos programas de modo desconectado. Essa percepção também foi manifestada pelo ex-Ministro do antigo MDA, Pepe Vargas, na 57a Reunião do CONDRAF, em 19 de fevereiro de 2014: "é sempre um desafio nós superarmos aquela cultura mais compartimentalizada, aquela cultura de cada ministério cuidando do seu mister [...] não é só interinstitucional no nosso plano federal. Ele também é interfederativo"11.

Ademais, diversos entrevistados afirmaram que, antes da extinção do MDA, havia uma atuação desse ministério no sentido de promover a articulação das ações, mas que após sua extinção, o nível de integração diminuiu ainda mais, diante do enfraquecimento do ator que tinha como responsabilidade capitanear o processo de valorização da agricultura familiar.

A literatura descreve situações que parecem encaixar-se bem com o panorama encontrado: para Mandell e Keast (2007), em redes com baixo nível de integração, os membros permanecem independentes entre si e interagem apenas quando necessário. As ligações entre eles são soltas e infrequentes. Segundo Peters (1998), seria o nível mínimo de integração, ou seja, aquele no qual as organizações são conscientes das atividades umas das outras e realizam esforços honestos para não prejudicar as demais e para não duplicar esforços, o que está longe de conseguir dar conta dos problemas enfrentados pelo setor público.

Diante disso, conclui-se que o desenho formatado para a rede, por meio das normas que definem as atribuições dos atores, prevê um arcabouço denso no centro e com flexibilidade nas margens. No entanto, na prática, mesmo esse núcleo duro tem atores com níveis variáveis de dedicação à rede, tanto quando se analisa ações de definição de diretrizes pelos ministérios, quanto quando se analisa a presença dos órgãos nos diferentes pontos do território.

Acredita-se que isso decorra em grande medida do fato de que diversos membros da rede têm em sua esfera de atuação uma miríade de outros temas que não são ligados à agricultura familiar. Assim, diante de variações nas preferências das lideranças ou da escassez de recursos, algumas vezes o tema da agricultura familiar será priorizado e em outras, não. Como Provan e Milward (1991) já destacaram: uma agência que provê majoritariamente serviços para outros públicos-alvo tende a estar ligada à rede apenas marginalmente. Assim, o excesso de atribuições legais diante das capacidades dos órgãos dificulta sua atuação efetiva em todos os temas sob sua responsabilidade.

\footnotetext{
${ }^{10}$ Dados disponíveis no SIOP.

${ }^{11}$ Conforme transcrição ipsis verbis.
} 


\section{CONCLUSÃO}

Para bem avaliar o resultado de políticas públicas, há que se conhecer de que modo essas políticas são geridas. Assim, para políticas em que ações coordenadas se mostram importantes para a obtenção de resultados, a análise de redes de políticas públicas constitui ferramenta adequada, pois auxilia na compreensão da estrutura e do contexto no qual as políticas operam.

Portanto, essa abordagem foi aplicada à Política Nacional de Agricultura Familiar para conhecer a composição de sua rede de atores e as características das relações estabelecidas por eles, com vistas a lançar luz em fatores que podem afetar o alcance dos resultados esperados pelo público-alvo.

Quanto à composição da rede, foi identificado um total de 76 organizações membros (Provan \& Milward, 1995) e 95 stakeholders externos (Mitchell et al., 1997). Observou-se uma rede bastante heterogênea, o que aumenta a legitimidade da política pública, desde que tais atores sejam de fato atuantes (Mitchell \& Shortell, 2000). Porém, quanto às organizações representantes da agricultura familiar, percebeu-se que tais representações não lograram ser suficientemente fortes, fato corroborado pelo processo de gradativo enfraquecimento e posterior extinção do CONDRAF.

Quanto às relações, identificou-se uma rede integrada em seu centro e flexível em suas margens, na qual um conjunto de atores-chave é significativo para a operacionalização de diversos programas, enquanto uma série de outros atores tem atuações mais dispersas e/ou localizadas.

A principal limitação da análise realizada foi a dificuldade de estimar objetivamente a intensidade da participação dos atores na rede. Pôde-se evidenciar a participação de todos os atores com atribuições legais nos programas, embora com relevantes indícios de frágil engajamento por parte de diversos deles. Vale lembrar que esse nível variável de comprometimento foi observado não só para atores às margens da estrutura de rede, mas também para atores do grupo central, cujas atribuições são cruciais para o bom andamento dos programas.

Assim, ligações fracas parecem ser frequentes e pervasivas, diante do que se argumenta que o distanciamento observado compromete o funcionamento efetivo de toda a rede e a obtenção dos resultados esperados da ação pública (O’Toole, 1997; Provan \& Milward, 1995).

Ademais, os governos estaduais se destacaram em diversas estatísticas da rede. Eles obtiveram altos graus de saída, participando de grande quantidade de programas; altos índices de page ranks, mostrando que suas relações não são apenas numerosas, mas também relevantes, além de terem se destacado nas medidas de centralidade, indicando sua importância e capacidade de influenciar o fluxo de recursos e informações (Burt, 1992). Diante disso, destaca-se a importância do pacto federativo para o funcionamento da política de agricultura familiar e sugere-se que os gestores subnacionais devem ter seus interesses e demandas considerados, de modo a promover o efetivo alcance dos resultados propostos para os programas voltados a esse público.

Avaliar o alcance de resultados de políticas públicas ignorando o contexto de sua formulação e de seu gerenciamento significa perder a oportunidade de identificar causas importantes para eventuais falhas de desempenho. Por outro lado, lançar luz sobre esse contexto pode significar a oportunidade de aprimorar o funcionamento das relações da rede, com potenciais efeitos positivos nos resultados da política pública.

No caso da agricultura familiar, a estrutura formada pela rede fornece pistas profícuas para a compreensão das dificuldades enfrentadas na entrega de serviços aos diversos grupos dessa categoria, cujas necessidades são tão críticas para temas como segurança alimentar e preservação ambiental.

Sem desconsiderar o impacto de diferenças regionais sobre o tema, defende-se que futuras pesquisas devam utilizar análises comparativas qualitativas (qualitative comparative analysis [QCA]) envolvendo redes locais efetivas e outras de pior desempenho, de modo a identificar boas práticas que possam ser replicadas e fomentadas para o benefício de toda a agricultura familiar. 


\section{REFERÊNCIAS}

Bardin, L. (2009). Análise de conteúdo. Lisboa: Ed. 70.

Barrat, A., Barthélemy, M., Pastor-Satorras, R., \& Vespignani, A. (2004). The architecture of complex weighted networks. Proceedings of the National Academy of Sciences, 101(11), 3747-3752.

Bastian, M., Heymann, S., \& Jacomy, M. (2009). Gephi: an open source software for exploring and manipulating networks. In Proceedings of International AAAI Conference on Web and Social Media, Palo Alto, CA.

Berardo, R. (2009). Generalized trust in multi-organizational policy arenas: studying its emergence from a network perspective. Political Research Quarterly, 62(1), 178-189.

Blondel, V. D., Guillaume, J. L., Lambiotte, R., \& Lefebvre, E. (2008). Fast unfolding of communities in large networks. Journal of Statistical Mechanics: Theory and Experiment, 10, 10008.

Borgatti, S. P. (2005). Centrality and network flow. Social Networks, 27(1), 55-71.

Borgatti, S. P., Everett, M., \& Johnson, J. (2013). Analyzing social networks. Thousand Oaks, CA: SAGE.

Brandes, U. (2001). A faster algorithm for betweenness centrality. The Journal of Mathematical Sociology, 25(2), 163-177.

Brin, S., \& Page, L. (1998). The anatomy of a large-scale hypertextual web search engine. Computer Networks and ISDN Systems, 30, 107-117.

Burt, R. S. (1992). Structural holes: the social structure of competition. Cambridge, MA: Harvard University Press.

Caume, D. J. (2009). Agricultura familiar e agronegócio: falsas antinomias. Redes, 14(1), 26-44.

Cazella, A. A., Capellesso, A. J., Medeiros, M., Tecchio, A., Sencébé, Y, \& Búrigo, F. L. (2016). Políticas públicas de desenvolvimento rural no Brasil: o dilema entre inclusão produtiva e assistência social. Política e Sociedade, 15(spe.), 49-79.

Cline, K. (2000). Defining the implementation problem: organizational management versus cooperation. Journal of Public Administration Research and Theory, 10(3), 551-571.

Da Ros, C. (2006). As políticas agrárias durante o governo Olívio Dutra e os embates sociais em torno da questão agrária gaúcha (19992002) (Tese de Doutorado). Universidade Federal Rural do Rio de Janeiro, Rio de Janeiro, RJ.

Decreto n. 9.186, de 1o de novembro de 2017. (2017). Dispõe sobre a composição, a estruturação, as competências e o funcionamento do Conselho Nacional de Desenvolvimento Rural Sustentável. Diário Oficial da União, Brasília, DF, 3 nov. 2017, Seção 1.

Dias, M. (2007). As mudancas de direcionamento da política nacional de assistência técnica e extensão rural (PNATER) face ao difusionismo. Oikós: Revista Brasileira de Economia Doméstica, 18(2), 11-21.

Ferlie, E., FitzGerald, L., McGivern, G., \& Dopson, S. (2011). Public policy networks and "wicked problems": a nascent solution? Public Administration, 89(2), 307-324.

Fernandes, A. H. M. (2016). A Frente Ampla da Agropecuária Brasileira na Constituinte de 1988: o patronato rural e o projeto de modernização conservadora do campo. In Anais do 17ํㅡ Encontro de História da ANPUH-RIO, Nova Iguaçu, RJ.

Fischer, M., \& Sciarini, P. (2015, July). Unpacking reputational power: intended and unintended determinants of the assessment of actors' power. Social Networks, 42, 60-71.

Grisa, C., \& Schneider, S. (2014). Três gerações de políticas públicas para a agricultura familiar e formas de interação entre sociedade e Estado no Brasil. Revista de Economia e Sociologia Rural, 52(supl. 1), 125-146.

Guanziroli, C. (1999). Reforma agrária e globalização da economia: o caso do Brasil. Brasília, DF: Instituto Nacional de Colonização e Reforma Agrária.

Jennings, E. (1994). Building Bridges in the Intergovernmental Arena: Coordinating Employment and Training Programs in the American States. Public Administration Review, 54(1), 52-60.

Jennings, E., \& Ewalt, J. (1998). Interorganization coordination, admistrative consolidation, and policy performance. Public Administration Review, 58(5), 417-428.

Lei n. 4.504, de 30 de novembro de 1964. (1964). Dispõe sobre o Estatuto da Terra, e dá outras providências. Diário Oficial da União, Brasília, DF, 30 nov. 1964, Seção 1.

Lei n. 8.629, de 25 de fevereiro de 1993. (1993). Dispõe sobre a regulamentação dos dispositivos constitucionais relativos à reforma agrária, previstos no Capítulo III, Título VII, da Constituição Federal. Diário Oficial da União, Brasília, DF, 26 fev. 1993, Seção 1.

Leifeld, P., \& Schneider, V. (2012). Information exchange in policy networks. American Journal of Political Science, 53(3), 731-744.

Mandell, M., \& Keast, R. (2007). Evaluating network arrangements: toward revised performance measures. Public Performance \& Management Review, 30(4), 574-597.

Mitchell, R., Agle, B., \& Wood, D. (1997). Toward a theory of stakeholder identification and salience: defining the principle of who and what really counts. The Academy of Management Review, 22(4), 853-886.

Mitchell, S., \& Shortell, S. (2000). The governance and management of effective community health partnerships: a typology for research, policy, and practice. The Milbank Quarterly, 78(2), 241-289.

Moreira, J. (2009). Uma visão do papel da agricultura familiar no Brasil. Novos Cadernos NAEA, 12(2), 57-88.

Navarro, Z. (2010). A agricultura familiar no Brasil: entre a política e as transformações da vida econômica. In J. G. Gasques, J. E. Vieira, Filho, \& Z. Navarro (Ed.), A agricultura brasileira: desempenho, desafios e perspectivas (pp. 185-211). Brasília, DF: Instituto de Pesquisa Econômica Aplicada.

O’Toole, L. (1997). Treating networks seriously: practical and researchbased agendas in public administration. Public Administration Review, 57(1), p. 45-52.

Peters, B. G. (1998). Managing horizontal government: the politics of coordination (Research Paper n. 21). Ottawa, Canada: Canadian Centre for Management Development. 
Picolotto, E. (2014). Os atores da construção da categoria agricultura familiar no Brasil. Revista de Economia e Sociologia Rural, 52(supl. 1), 63-84.

Pressman, J., \& Wildavsky, A. (1984). Implementation: how great expectations in Washington are dashed in Oakland (3a ed.). Los Angeles, CA: University of California Press.

Provan, K., \& Kenis, P. (2007). Modes of network governance: structure, management, and effectiveness. Journal of Public Administration Research and Theory, 18(2), 229-252.

Provan, K., \& Lemaire, R. (2012). Core concepts and key ideas for understanding public sector organizational networks: Using research to inform scholarship and practice. Public Administration Review, 72(5), 638-648.

Provan, K., \& Milward, H. (1991). Institutional-Level Norms and Organizational Involvement in a Service-Implementation Network. Journal of Public Administration Research and Theory, 1(4), 391-417.
Provan, K., \& Milward, H. (1995). A preliminary theory of interorganizational network effectiveness: a comparative study of four community mental health systems. Administrative Science Quarterly, 40(1), 1-33.

Raab, J., Mannak, R., \& Cambré, B. (2013). Combining structure, governance, and context: a configurational approach to network effectiveness. Journal of Public Administration Research and Theory, 25(2), 479-511.

Repetto, F. (2009). Coordinación y gestión pública integral en América Latina: notas conceptuales. In F. Repetto, H. M. S. Almeida, J. Toni, M. S. Fonseca, M. J. M. Cabello, O. O. Aguilar ... J. C. Vaz (Ed.), Reflexões para Ibero-America: planejamento estratégico (pp. 11-20). Brasília, DF: Escola Nacional de Administração Pública.

The Gephi Consortium. (c2017). Gephi: The Open Graph Viz Platform. Recuperado de https://gephi.org/

Luciana Nunes Goulart

ORCID: https://orcid.org/0000-0003-4112-8775

Mestre em Administração pelo Programa de Pós-Graduação em Administração da Universidade de Brasília (PPGA/UnB); Auditora Federal de Controle Externo do Tribunal de Contas da União. E-mail: lucianagoulart33@gmail.com

Diego Mota Vieira

ORCID: https://orcid.org/0000-0003-1275-1648

Doutor em Administração pelo Programa de Pós-Graduação em Administração da Universidade de Brasília (PPGA/UnB); Professor Adjunto no Programa de Pós-Graduação em Administração da Universidade de Brasília (PPGA/UnB). E-mail: diego1mv@yahoo.com.br

Daniela Matias de Carvalho Bittencourt ORCID: https://orcid.org/0000-0002-3137-6567

Doutora em Ciências Biológicas pela Universidade de Brasília; Pós-Doutora por La Recherche Agronomique pour le Développement, CIRAD, França; Pesquisadora da Embrapa Recursos Genéticos e Biotecnologia. E-mail: daniela.bittecourt@embrapa.br 